\title{
Dynamic Order Acceptance and Capacity Planning on a Single Bottleneck Resource
}

\author{
Jade Herbots • Willy Herroelen • Roel Leus* \\ Department of Decision Sciences and Information Management, \\ Katholieke Universiteit Leuven, Belgium \\ \{Jade.Herbots ; Willy.Herroelen ; Roel.Leus\}@econ.kuleuven.be
}

We present a tactical decision model for order acceptance and capacity planning that maximizes the expected profits from accepted orders, allowing for aggregate regular as well as non-regular capacity. The stream of incoming order arrivals is the main source of uncertainty in dynamic order acceptance and the company only has forecasts of the main properties of the future incoming projects. Project proposals arrive sequentially with deterministic interarrival times and a decision on order acceptance and capacity planning needs to be made each time a proposal arrives and its project characteristics are revealed. We apply stochastic dynamic programming to determine a profit threshold for the accept/reject decision as well as to deterministically allocate a single bottleneck resource to the accepted projects, both with an eye on maximizing the expected revenues within the problem horizon. We derive a number of managerial insights based on an analysis of the influence of project and environmental characteristics on optimal project selection and aggregate capacity usage.

Keywords: order acceptance, capacity planning, multi-project, stochastic dynamic programming.

\section{Introduction}

Many companies tend to accept all projects with a positive net present value (NPV), without consideration of the effect on the planning of the already accepted projects. In case of external projects (projects performed for customers external to the organization), this is often the consequence of the functional separation between the order-acceptance decision, which is made by the sales department, and capacity planning, which usually lies in the hands of the production department. These two departments generally have conflicting objectives: in order to boost sales, sales departments try to accept as many projects as possible, while production attempts to live up to promised delivery dates. This divergence of interests can result in considerable delays, violated due dates and/or excessive use of highly expensive

\footnotetext{
${ }^{*}$ Corresponding author
} 
non-regular capacity such as overtime and temporary labor. It is therefore essential that project selection and planning be integrated [52].

This paper examines the simultaneous dynamic order-acceptance and capacity-planning decision. Order acceptance refers to the accept/reject decision an over-demanded company has to make upon project arrival. Capacity planning is concerned with making a rough sketch of the aggregate resource usage (regular and non-regular) and the timing of the work packages of both accepted as well as candidate projects.

In a multi-project environment, projects typically share common resources. Adequate management of these scarce resources is therefore of crucial importance. Consequently, the development of good acceptance rules and capacity-planning tools is extremely relevant, as they can support decisions such as due-date quotation, price quotation and hiring non-regular capacity. Appropriate order acceptance and capacity planning allows to gain a larger control over the use of non-regular capacity and increase profits. Since the quoted completion times are compatible with the available capacity, operational scheduling becomes less troublesome. This leads to more reliable due dates, which improves the delivery performance and creates a competitive advantage to the company. These benefits constitute the motivation for this research.

Most of the existing literature on order acceptance and capacity planning deals with static models, in which project selection is performed only once, at the beginning of the planning horizon. Although some models also consider the possibility of intermediate action, they are mainly suitable for internal project selection, where the set of projects available for execution during the planning horizon is known in advance. By internal projects, we refer to projects that have been proposed by internal customers, examples are internal R\&D (Research and Development) or NPD (New Product Development) projects. As a consequence, the static approach is less realistic when dealing with external projects which, in general, arise dynamically to the organization and require immediate response. Models specific to this situation are called dynamic models. This paper is concerned with the development of a dynamic model for dealing with external project arrivals.

The issue of project selection can be positioned at the tactical decision-making level and is part of project portfolio management, which is concerned with project selection and prioritization by executive and senior management, with a focus on strategic medium- and long-term decisions. If the financial implications of individual projects have a considerable impact on the vitality of the organization, project selection is of strategic importance. 
Strategic decision making is concerned with setting global capacity levels, the location of new sites, etc. At the tactical level, we encounter problems such as selecting non-strategic projects, aggregate capacity planning, due-date setting, order bidding, etc. The lowest decision level is the operational level, where detailed scheduling and resource-allocation problems are tackled.

In this paper, we present a tactical decision model for order acceptance and capacity planning that maximizes the expected profits from accepted orders. Capacity is considered at an aggregate level, while the regular per-period capacity is limited. Additionally, non-regular capacity units can be allocated at specific per-unit costs. At the completion of a project, the company receives a payoff, which it can reinvest until the end of the problem horizon at a specified interest rate. The stream of incoming order arrivals is the main source of uncertainty in dynamic order acceptance and the company only has forecasts of the main properties of the future incoming projects. Project proposals arrive sequentially with deterministic interarrival times and a decision on order acceptance and capacity planning needs to be made each time a proposal arrives. At the moment of arrival, detailed problem characteristics are not yet available; nevertheless, the company has information at its disposal on the main project characteristics, that is to say revenue, workload and due date. Precedence relations and non-preemption constraints may be imposed between the work packages that make up the workload.

Our model is particularly relevant for environments in which a scarce resource acts as a single static bottleneck and where at least rudimentary information about the work content of the proposed and future projects is available. Examples of such scarce resources are e.g. a shipyard, a testing lab, a fabrication line and a specialized engineer. When these resources become bottlenecks, their allocation has a major influence on profitability [1]. Examples of such environments are MTOs (manufacture-to-order) with a single static bottleneck resource (e.g. a semiconductor fabrication line producing a mix of products [41]), construction environments and maintenance projects (e.g. in the avionics industry and shipyards, where aircraft or ships are scheduled for preventive maintenance projects [13]).

In order to tackle the dynamic order-acceptance and capacity-planning problem at hand, we have interpreted it as an extension of the optimal stopping problem (e.g. Bertsekas [8]). This approach allows us to formulate a stochastic dynamic program (SDP) that maximizes the company's long-run expected profits, contrary to traditional static tactical capacityplanning models (e.g. [13, 24]), which aim to maximize the immediate reward from accepting 
an order. The latter approach corresponds to the classic NPV-rule, which ignores future opportunities, and is therefore overly conservative (see e.g. [17], [38]).

The contributions of this text are the following. First, we introduce a formal problem statement that relates the problem characteristics and the basic elements of the SDP. Next, we present our SDP that determines a profit threshold for the accept/reject decision as well as the capacity allocation maximizing the expected revenues within the problem horizon. We also define a number of dominance rules that narrow the way in which capacity may be allocated. We derive a number of managerial insights based on an analysis of the influence of project and environmental characteristics on optimal project selection and aggregate capacity usage. For example, we determine the circumstances under which 'short-cut' planning rules exist. In case of reinvestment revenues, we quote a cut-off value that determines the planning rule. Finally, we quantify the value of non-regular capacity units and due-date flexibility.

The remainder of this paper is organized as follows. Section 2 contains an overview of the literature on project selection, in which we discuss both static and dynamic models. In Section 3, we introduce the basic problem characteristics and give an extended problem description. Section 4 contains a presentation of our SDP model. In Section 5, we use the developed model to derive a number of important insights. For instance, we quantify the value of capacity and due-date flexibility and we incorporate tardiness penalties. SDP also suffers from the curse of dimensionality (term suggested by Bellman [7]), and in our analysis we examine under which circumstances efficient 'short-cut' planning rules are helpful. Finally, in Section 6, we draw some conclusions and look at future research opportunities.

\section{$2 \quad$ Literature survey}

Project selection has been studied in a broad variety of research domains, among which operations management, finance and managerial economics. In this section we discuss the relevant literature for the static and dynamic selection problem primarily in operations management but also in the other two cited literature streams. Several exact and approximate selection and planning methods have been proposed for the static problem; this literature is the topic of Section 2.1. As for the dynamic context, the existing work is relatively scarce and will be discussed in Section 2.2. 


\subsection{The static selection problem}

Static project selection implies the optimization of qualitative factors (e.g., alignment with company strategy), quantitative criteria (e.g., return on investment, NPV) or a combination of both. Especially for strategic decision making, the objectives will often include qualitative factors in addition to merely quantitative profitability measures. A large part of the literature is dedicated to $R \& D$ environments. In $R \& D$, the encountered decision problems are mostly strategic, given that effective R\&D portfolio management is a prerequisite for the medium and long-term success of technology-driven organizations [10]. Baker and Freeland [3], Hall and Nauda [23] and Henriksen and Traynor [25] present surveys of R\&D project selection. Henriksen and Traynor [25] categorize a wide range of tools with varying metrics and selection methods. The following paragraphs provide a brief overview of the most-employed methods, namely mathematical programming, scoring and sorting methods, financial models and mapping.

Early attempts to tackle static selection usually took the form of mathematical-programming models related to knapsack formulations, for an overview we refer to [48]. Static selection is regarded here as the evaluation of a set of candidate projects, where the goal is to select a subset of projects that maximizes some objective function without violating

the constraints. Articles [5], [6] and [22] use integer-programming formulations. Some more extended models take payoff interactions between projects into account (interdependencies between the financial benefits $[16,20]$ ) as well as technical interactions (overlap between project contents [11]). The observation that mathematical-programming tools have not found widespread acceptance in practice has been confirmed recently by Loch et al. [39]. Baker and Freeland [3] and Hall and Nauda [23] assess why few quantitative models for R\&D project selection and capacity allocation have been implemented by managers.

Other ways of approaching the static selection problem are scoring and sorting models. These models evaluate projects based on financial or non-financial measures. Projects are ranked via a score determined by e.g. analytical hierarchy process (AHP), see [9, 45]. Other scoring methods were developed in [19] and [25].

Financial models for portfolio selection often start from the Markowitz model, which minimizes the variability of the return of a portfolio subject to bounds on the expected return, see e.g. [40]. Application of this model to R\&D portfolio selection has been suggested in the literature $[20,42]$. In his dissertation, Jørgensen [28] gives an extensive overview of 
the literature on financial methods applied to project selection.

Yet another angle to approach the portfolio selection problem can be found in mapping techniques. These are graphical and charting techniques that evaluate qualitative measures by visualizing the balance of the portfolio. Most of this literature descends from the disciplines of strategy and marketing. Wheelwright and Clark [50] give practical advice on how to organize the product development process.

The foregoing techniques mainly apply to strategic decisions. At the tactical decision level we encounter the so-called Rough-Cut Capacity Planning (RCCP) problem, which is a specific type of tactical capacity planning. Within RCCP, work packages can be executed at a variable intensity $[13,24]$. These mathematical-programming models minimize the nonregular capacity and/or tardiness costs; they can serve as a basis for heuristics [21]. Kis [31] and Kolish and Meyer [34] model the problem of selecting and planning projects as extensions of the resource-constrained project scheduling problem (RCPSP). These mathematicalprogramming models can be incorporated in project selection decisions to assess the impact of candidate orders on the capacity profile and to determine a reliable due date. Although these models are able to deal with a high problem complexity, they are static models and only consider immediate rewards (application of the NPV-rule), not taking opportunities from future arriving offers into account. Loch and Bode-Greuel [38] demonstrate, albeit in a somewhat different setting (i.e. R\&D environment), that all future opportunities should be recognized in case of managerial flexibility (in our case, this is the possibility to reject an offer and to wait for a better one to arrive before allocating capacity). In this regard, we develop a dynamic model that incorporates the potential future rewards into the current decision.

Detailed operational scheduling, relating to even shorter time horizons and higher planning frequencies than tactical models, is performed at the operational decision level [15]. An example of operational project selection can be found in [34] and [51]. Within the operational domain of job-shop planning, job selection has been a topic of growing interest in the last decade; for examples we refer to [12] and [46], in which a pool of orders is considered and sequencing and order acceptance are separated. In [36], these models are extended to multiple periods. In our opinion, the general lack of information when a project is initially presented to a company makes such methods unfeasible for practical multi-project planning. 


\subsection{The dynamic selection problem}

Dynamic project selection has been studied to a lesser extent than its static counterpart. Nevertheless, a broad variety of solution methods has been proposed.

A basic approach to dynamic selection can be found in the dynamic stochastic knapsack model developed by Kleywegt and Papastavrou [32, 33]. This problem is an extension of the stochastic knapsack problem in [43] to the case where items arrive over time with an unknown size and reward.

Queueing approaches to multi-project planning were introduced by Adler et al. [1] and Levy and Globerson [35]. The NPD process is modelled as a stochastic processing network in which engineering resources are 'workstations' and projects are 'jobs' that flow between the workstations. Both sources analyze the crucial issues responsible for time delays and cost overruns. De Reyck [14] points out that the resulting analysis will not produce any detailed scheduling information on when to initiate or terminate individual activities or entire projects, but only allows for estimation of the average time spent on a single project. In [29] and [37] the dynamic selection problem is treated as an admission control problem, a known problem within queueing theory.

Recently, Ebben et al. [18] have used simulation to compare different order-acceptance strategies in a generic job-shop. A similar method was proposed in [2] and [49] for productionto-order environments, together with heuristics for scheduling the accepted work orders. In a completely different context, Balakrishnan et al. [4] implement a decision-theory-based approach that reserves parts of the capacity for specified order types through a capacity allocation policy.

Most similar to the model described in this text is the work of Perry and Hartman [41], who examine the problem of selecting a number of orders with fixed production schemes from a set of arriving project offers. They solve a specific multi-knapsack problem through SDP. The suggested method determines the order combination that maximizes the expected future profits. Their model, however, does not define exact acceptance criteria nor does it allow the planner to deviate from the fixed production schemes. Moreover, the allocation of non-regular capacity units is not considered. 


\section{Problem description}

In this section we present a detailed problem statement. We introduce the major characteristics of an incoming project, namely its revenue, due date and workload, and discuss the incorporation of precedence relations and non-preemption constraints. We describe our assumptions regarding the order-arrival process and we elaborate how capacity utilization is modelled. The modelling choices have been made with an eye on the development of the SDP in Section 4; we discuss how these assumptions relate to a practical multi-project setting.

\subsection{Project characteristics}

Project acceptance and capacity planning is confronted with restricted resource availability. We express both the project workload as well as the aggregate capacity available in the organization as a number of capacity units (e.g. man-hours). We refer to each individual capacity unit belonging to the work content of a project as a work package.

It is standard practice for project management to structure the work content into small manageable elements as a result of the development of a work breakdown structure (WBS). A WBS is a product-oriented family-tree subdivision of the hardware, services and data required for project realization, which provides a common framework for breaking the work down into work packages, thus providing a greater probability that every major and minor activity will be accounted for [30].

In our model, each project $k$ constitutes an aggregated workload on a static bottleneck resource, expressed as a number $p_{k}$ of work packages. Precedence relations may apply between the work packages (e.g. a testing department that needs to perform subsequent tests) as well as non-preemption constraints. In case of order acceptance, all work packages must be deterministically planned between the release time $r_{k}$ and the due date $d_{k}$, which is regarded here as a deadline. This means that due dates cannot be exceeded and orders for which the due date cannot be met, must be rejected. In Section 5.7, we discuss the possibility of due-date violation at a tardiness penalty. The payoff of a work order, denoted as $y_{k}$, is generated immediately when the work is completed, this is when all work packages have been executed according to the capacity plan. We assume that all these revenues can be reinvested at a fixed interest rate $i \geq 0$. 


\subsection{The order arrival process}

A Request for Proposal (referred to as RFP) is an invitation for suppliers, through a bidding process, to bid on a specific product or service. An RFP typically involves more than the price, so, in this article, it is more appropriate to use the term Request for Quotation (RFQ), where discussions are not required with bidders (mainly when the specifications of a product or service are already known), and price is the main or only factor in selecting the successful bidder. In what follows, we use the terms 'order' and 'project' to refer either to an RFQ or to a request for execution of an order at a given price. Since we establish a profit threshold below which prices lead to rejection, price setting and order acceptance at a fixed price can be treated similarly. In both cases, we assume that order acceptance results in a fixed-price contract (see e.g. [27]). Our model is developed from the viewpoint of one individual bidder, and decisions are made without consideration of competitors.

The order arrivals represent the main source of uncertainty in our dynamic order-acceptance model. When a company has to make an accept/reject decision, it has at its disposal only rudimentary information about the project in question and forecasts of the main characteristics of the future incoming projects (e.g. based on sales-force polling). To describe the order arrival process, we first discretize the planning horizon into $T$ periods or time buckets (e.g. weeks or months) of equal length. Additionally, we introduce the concept of a stage, which is the time interval between two consecutive project arrivals: a new stage starts every time a new project arrives. The number of projects arriving sequentially within the planning horizon $T$ (and hence, the number of stages) is $N$ and we assume that the arrivals have equal interarrival times. The properties of the order arrival process correspond with those for the optimal stopping problem that was the basis for our SDP and, consequently, are essential for the applicability of the algorithm proposed in Section 4.

Although the use of deterministic interarrival times and a fixed number of arrivals may seem restrictive, it can be interpreted as a representation of the fact that we use forecasts of the future arrival stream, for which only an average interarrival time is known. When this leads to non-discrete time instants, the planning decisions for each project are shifted towards the start of the next period. Unequal time intervals are easily incorporated, but would not really add to the value of the results of the current text. Stochastic interarrival times can be approximated by increasing the number of stages within the fixed time horizon and adapting the probability distribution of the project characteristics. A visualization of 


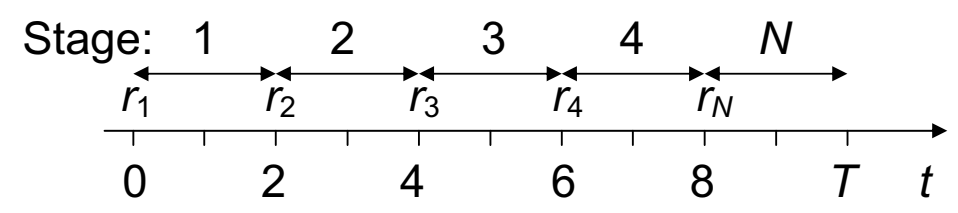

Figure 1: Order arrival process

the order arrival process is given in Figure 1 with $T=10$ and $N=5$. At the start of each stage $k, k=1, \ldots, N$, we decide upon order acceptance and capacity allocation of project $k$, with stage 1 being the first stage. Project $k$ can be planned from its release time $r_{k}$.

The main characteristics of a project proposal $k$ are its payoff $y_{k}$, a positive workload, consisting of $p_{k}$ work packages and a due date $d_{k}$. From a given positive maximum time lag $l_{k}$ allowed for realizing the project, one can easily derive the due date by adding the stage's release time $r_{k}$ to the maximum time lag: $d_{k}=r_{k}+l_{k}$. The maximum time lag expresses the maximum time, a customer would accept to wait for the realization of his project. If the firm is unable to quote a completion time within this lag, the customer will turn to the firm's competitors, irrespective of the firm's conditions. We represent the arriving offers as $\mathbf{w}_{1}, \mathbf{w}_{2}, \ldots, \mathbf{w}_{N}$, with $\mathbf{w}_{k}=\left(p_{k}, y_{k}, l_{k}\right)$, for stage $k=1, \ldots, N$. A decision needs to be made regarding order $\mathbf{w}_{1}$, and estimates about the characteristics of the stream of future order arrivals are captured as follows: values $p_{k}, y_{k}$ and $l_{k}$ are assumed to be realizations of $P, Y$ and $L$, respectively, each of which is a random variable. Hence, the values $\mathbf{w}_{k}, k=2, \ldots, N$, are independent realizations of the multivariate random variable $\mathbf{W}=(P, Y, L)$. The support of $P$ and $L$ only contains natural numbers.

\subsection{Capacity profile}

In this paper, we consider only one resource type, which is taken to represent the bottleneck resource of the company. For R\&D projects, for instance, this resource could be a critical testing equipment or the allocated periodical budget, while in an MTO environment it might represent a single machine or a team of engineers. The company owns a limited number of aggregate bottleneck capacity units. The amount of regular capacity units is the result of a long-term strategic decision that cannot be revised within the time horizon considered in our planning framework. In contrast, the number of non-regular capacity units can be altered as a result of working overtime, hiring temporary labor or outsourcing.

We count the available regular and non-regular capacity units in every time period by 


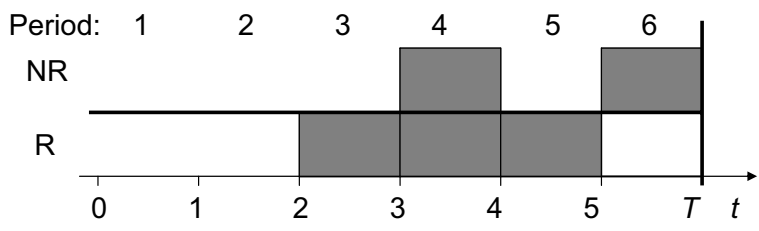

Figure 2: Capacity profile with regular (R) and non-regular (NR) capacity units means of a capacity profile, which is a vector

$$
\mathbf{x}_{k}=\left(\mathbf{m}_{k}, \mathbf{s}_{k}\right), \text { with }\left\{\begin{array}{l}
\mathbf{m}_{k}=\left(m_{r_{k}+1}, \ldots, m_{T}\right) \\
\mathbf{s}_{k}=\left(s_{r_{k}+1}, \ldots, s_{T}\right)
\end{array}\right.
$$

where $k$ represents the stage number. Here $m_{t}$ is the number of available regular capacity units in time period $t$ and $s_{t}$ the maximum number of non-regular capacity units that can be hired during time period $t$. The cost per unit of consumed non-regular capacity is $c$, whereas the actual utilization of regular capacity does not give rise to incremental costs. In stage $k, \mathbf{x}_{k}$ only reflects resource availability from time $r_{k}$ onwards since this vector contains all information relevant for making decisions regarding offer $k$. Implicitly, this derives from the fact that all unused capacity units before $r_{k}$ have 'perished'. In the remainder of this article, we will speak of perishable resources (cfr. [47]); the scheduling literature sometimes uses the term renewable resources (see, for instance [15]).

An illustration is provided in Figure 2. The capacity profile for the first stage, starting at time 0 , is $\mathbf{x}_{1}=\left(\mathbf{m}_{1}, \mathbf{s}_{1}\right)=((0,0,1,1,1,0),(0,0,0,1,0,1))$. Remark that even the capacity profile in stage 1 can exhibit an uneven pattern: earlier decisions, e.g. under the form of firm planned orders, may already have allocated capacity units in the current planning horizon.

\subsection{The order plan}

Upon arrival, the organization can choose whether to reject or to accept the project according to any eligible order plan. An order plan is an allocation of capacity units to the different work packages of a project. We represent an order plan $j$ for arrival $k$ as a vector $\mathbf{a}_{k}^{j}=$ $\left(\mathbf{a}_{k m}^{j}, \mathbf{a}_{k s}^{j}\right)$, where $\mathbf{a}_{k m}^{j}$ and $\mathbf{a}_{k s}^{j}$ have the same dimension as $\mathbf{m}_{k}$ and $\mathbf{s}_{k}$, and count the number of (regular and non-regular) capacity units that are allocated to project $k$ in each relevant time period.

We define an order plan to be feasible if two conditions are fulfilled: (1) the total workload of the project is covered; and (2) all work packages are planned between the stage's release time $r_{k}$ and the project's deadline $d_{k}$. The set of feasible order plans in stage $k$ is denoted 
as $\mathcal{F}_{k}$. Additionally, the set of order plans to be examined may be reduced because of practical considerations, which may take the form of precedence relations between the work packages or non-preemption constraints. It may be the case, for instance, that a complete order is imposed on the work packages, which is the case for e.g. stage-gate development processes (see [10]), or that sequential testing procedures need to take place in consecutive time periods.

When an offer is accepted, its capacity allocation is simultaneously determined by the SDP, in such a way that the expected total profits are maximized. In case an exceptional opportunity presents itself, or when actual resource usages differ from the the planned amounts, the decision maker may decide to replan. Such a replanning decision has no consequences for subsequent order acceptance decisions, which can be based on updated capacity profiles. In case the company rejects the offer, no capacity is reserved and no further action is taken until the next project arrival. This rejection cannot be withdrawn. We associate a 'degenerate' order plan $\mathbf{a}_{k}^{0}=\mathbf{0}$ (the null vector) with rejection, and we let symbol $\mathcal{A}_{k}$ represent the set of all eligible order plans augmented with $\mathbf{a}_{k}^{0}$.

\section{Stochastic dynamic programming}

We present an SDP approach (see, for instance [44]) for order acceptance and capacity planning. The problem is modelled as an extension of the optimal stopping problem (e.g. Bertsekas [8]) for which we regard the capacity units as perishable assets. By selling the assets, individually or in group, at the highest expected offer, we maximize the expected profits. This interpretation of the problem will allow us to determine an optimal threshold and order plan for every arriving offer.

The basic optimal stopping problem is discussed in Section 4.1. We extend this problem in Section 4.2, and provide some illustrations.

\subsection{Optimal stopping problem}

We investigate and extend one specific variant of the optimal stopping problem, namely the asset selling problem as described by Bertsekas [8]. In this setting, an asset seller receives a random bid $w_{k}$ in each period $k$, over a horizon of $N$ periods. If the person accepts the offer, he or she receives the payoff which can be reinvested at an interest rate $i$. 
Bertsekas presents an SDP to determine an optimal threshold in every period for accepting a bid. Its elements are the following: the state of the system in each stage $k$, represented by $x_{k}$, and the control space. If the bid is accepted, the system goes into the termination state, otherwise the state equals the last considered bid. The control space contains the possible actions we can undertake when arriving in a new stage, which is at the arrival of a new bid. There are two possible actions $a_{k}$, namely $a_{k}^{0}$ : the rejection of the bid, and $a_{k}^{1}$ : the sale of the asset.

\subsection{Dynamic order acceptance and planning}

This section provides a detailed description of our solution approach for dynamic order acceptance and planning. We first present our stochastic dynamic program, next we illustrate the approach with two examples.

\subsubsection{Stochastic dynamic program}

Based on the previously discussed optimal stopping problem, we develop an SDP consisting of $N$ stages, where $N$ equals the number of offers within the planning horizon $T$. The arrival of an order corresponds to the beginning of a new stage $k$. The state in stage $k$ is the capacity profile $\mathbf{x}_{k}$. To alleviate the notation, we omit the perished periods from the state vector (which was also suggested in Section 3.3). To this aim we define a perishing function $v$ that transforms a vector $\mathbf{x}_{k}$ into a vector $\mathbf{x}_{k+1}$ from which the perished capacity units are removed. For the capacity profile in Section 3.3, we have $v\left(\mathbf{x}_{1}\right)=\mathbf{x}_{2}=((0,1,1,1,0),(0,0,1,0,1))$. We call a specific capacity unit current if it perishes in the following stage and future if not.

The control space $\mathcal{A}_{k}$ of the SDP consists of a variable number of possible actions, each corresponding with an order plan $\mathbf{a}_{k}^{j}$. The reward in stage $k$ for order plan $\mathbf{a}_{k}^{j}$ and offer $\mathbf{w}_{k}$ is determined as:

$$
g_{k}\left(\mathbf{a}_{k}^{j}, \mathbf{w}_{k}\right)= \begin{cases}0 & \text { if } j=0, \\ y_{k}(1+i)^{\left(T-t_{j}^{*}\right)}-c s_{j}^{*} & \text { otherwise }\end{cases}
$$

where $s_{j}^{*}$ denotes the number of non-regular capacity units employed when implementing order plan $\mathbf{a}_{k}^{j}$. Value $t_{j}^{*}$ is the realization time of an accepted project planned according to $\mathbf{a}_{k}^{j}$, and corresponds with the pay-out time of the project. As a result, $T-t_{j}^{*}$ is the period for which the company receives additional interest revenues. 
The backward SDP algorithm consists of iteratively solving the following recursion:

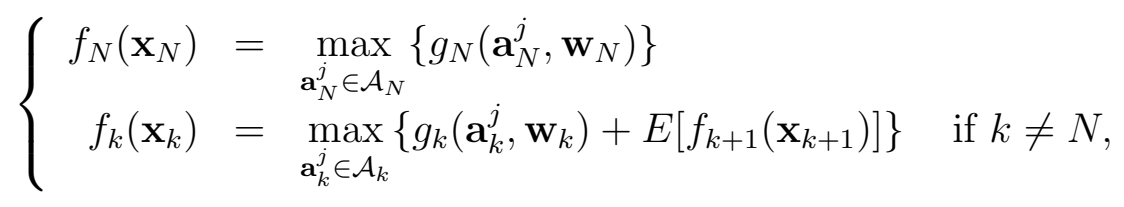

with

$$
\mathbf{x}_{k+1}=v\left(\mathbf{x}_{k}-\mathbf{a}_{k}^{j}\right)
$$

and $E[\cdot]$ the expectation operator. In Eq. (3), $f_{k}\left(\mathbf{x}_{k}\right)$ is the maximum expected reward that can be earned during stages $k, k+1, \ldots, N$ given that the initial state corresponds with $\mathbf{x}_{k}$. Eq. (4) describes the transition function that transforms $\mathbf{x}_{k}$ into $\mathbf{x}_{k+1}$, where the latter quantity represents the state or capacity profile after implementing order plan $\mathbf{a}_{k}^{j}$; if $j=0$, $\mathbf{x}_{k+1}$ equals $v\left(\mathbf{x}_{k}\right)$.

Definition 1. In stage $k$, an order plan $\mathbf{a}_{k}^{\tilde{j}}$ is dominated by another order plan $\mathbf{a}_{k}^{j}$ if

$$
\begin{array}{cl}
g_{N}\left(\mathbf{a}_{N}^{j}, \mathbf{w}_{N}\right) \geq g_{N}\left(\mathbf{a}_{N}^{\tilde{j}}, \mathbf{w}_{N}\right) & \text { if } k=N, \\
g_{k}\left(\mathbf{a}_{k}^{j}, \mathbf{w}_{k}\right)+E\left[f_{k+1}\left(\mathbf{x}_{k+1}\right)\right] \geq g_{k}\left(\mathbf{a}_{k}^{\tilde{j}}, \mathbf{w}_{k}\right)+E\left[f_{k+1}\left(v\left(\mathbf{x}_{k}-\mathbf{a}_{k}^{\tilde{j}}\right)\right)\right] & \text { if } k \neq N .
\end{array}
$$

Intuitively, one order plan dominates another if the second does not result in a larger value for $f_{k}\left(\mathbf{x}_{k}\right)$ according to Eq. (3). Lemmas 1, 3 and 4 below describe a number of situations in which dominated order plans can be recognized.

Lemma 1. An order plan which employs non-regular capacity units that can be replaced or partially replaced by regular capacity units from the same time period is always dominated by an order plan which has adopted these replacements.

The proof can be found in [26].

Definition 2. A state $\mathbf{x}_{k}$ is larger than $\left(>_{l}\right)$ another state $\tilde{\mathbf{x}}_{k}$ if the capacity profile of $\mathbf{x}_{k}$ contains every capacity unit of the capacity profile of $\tilde{\mathbf{x}}_{k}$ and at least one additional capacity unit. Two states are equal (=) if their stage number and capacity profile are the same.

Lemma 2. If $\mathbf{x}_{k}$ is larger than $\left(>_{l}\right) \tilde{\mathbf{x}}_{k}$ then the maximum expected reward from $\mathbf{x}_{k}$ is larger than or equal to the maximum expected reward from $\tilde{\mathbf{x}}_{k}$.

The proof can be found in [26]. 


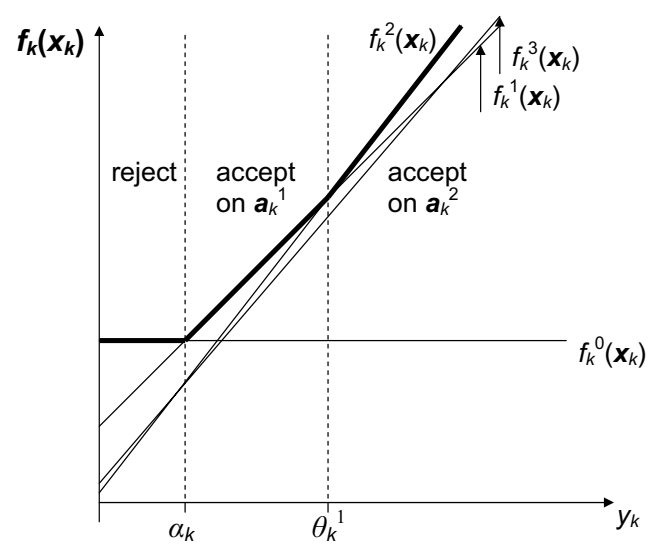

Figure 3: Threshold and order plan determination

Lemma 3. An order plan that results in a non-positive reward is always dominated by rejecting the offer.

The proof can be found in [26].

Lemma 4. An order plan $\mathbf{a}_{k}^{j}$ that employs one or more current regular capacity units dominates any order plan $\mathbf{a}_{k}^{\tilde{j}}$ that has replaced one or more of these current regular capacity units with future capacity units.

The proof can be found in [26]. Under certain conditions, the lemma can be strengthened.

Corollary 1. When $\mathcal{A}_{k}=\mathcal{F}_{k} \cup\left\{\mathbf{a}_{k}^{0}\right\}$, any order plan that allocates future capacity units while leaving current regular capacity units unallocated, is dominated.

The proof can be found in [26]. By iterative solution of the SDP recursion given by Eq. (3) we can derive the optimal acceptance threshold $\alpha_{k}$ and the optimal planning method for incoming order $\mathbf{w}_{k}$. To this aim we define $f_{k}^{j}\left(\mathbf{x}_{k}\right)$ as $g_{k}\left(\mathbf{a}_{k}^{j}, \mathbf{w}_{k}\right)+E\left[f_{k+1}^{j}\left(v\left(\mathbf{x}_{k}-\mathbf{a}_{k}^{j}\right)\right)\right]$ if $k \neq N$ and as $g_{N}\left(\mathbf{a}_{N}^{j}, \mathbf{w}_{N}\right)$ if $k=N . f_{k}^{j}\left(\mathbf{x}_{k}\right)$ has slope 0 if $j=0$ and slope $(1+i)^{\left(T-t_{j}^{*}\right)} \geq 1$ otherwise. The non-decreasing piecewise linear function $f_{k}\left(\mathbf{x}_{k}\right)$ can now be written as $\max _{\mathbf{a}_{k}^{j} \in \mathcal{A}_{k}}\left\{f_{k}^{j}\left(\mathbf{x}_{k}\right)\right\}$. In each of the subintervals $\left[0, \alpha_{k}\left[,\left[\alpha_{k}, \theta_{k}^{1}\left[,\left[\theta_{k}^{1}, \theta_{k}^{2}\left[, \ldots,\left[\theta_{k}^{n}, \infty\left[\right.\right.\right.\right.\right.\right.\right.\right.$ of $y_{k}$, the function $f_{k}\left(\mathbf{x}_{k}\right)$ coincides with one of the functions $f_{k}^{j}\left(\mathbf{x}_{k}\right)$. The threshold $\alpha_{k}$ is $\max \left\{y_{k} \mid f_{k}^{0}\left(\mathbf{x}_{k}\right) \geq f_{k}^{j}\left(\mathbf{x}_{k}\right), j \neq 0\right\}$. For each subinterval, the best order plan is the one that maximizes $f_{k}\left(\mathbf{x}_{k}\right)$ in that interval, as visualized in Figure 3. 


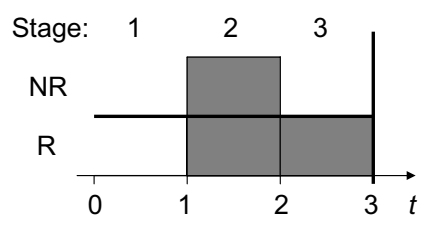

Figure 4: Example with regular (R) and non-regular (NR) capacity units

Observation 1. The maximum expected reward that can be earned during stages $k, k+$ $1, \ldots, N$ for a given initial state $\mathbf{x}_{k}, f_{k}\left(\mathbf{x}_{k}\right)$, is a non-decreasing convex function of the project payoff $y_{k}$.

\subsubsection{Example for single-sized orders and infinite maximum time lags}

This first example considers only project proposals with workloads equal to one work package and infinite deadlines. We consider a company that has been offered a project with a payoff of 10 if realized with regular capacity $\left(y_{1}=10\right)$. Assume we are at time instant 0 looking at the next three months $(T=3)$. The capacity profile within the problem horizon is $\mathbf{x}_{1}=((0,1,1),(0,1,0))$ and is depicted in Figure 4 . We ignore the possibility to reinvest the revenues and set the interest rate $i$ to 0 . The cost of one unit of non-regular capacity is $c=5$. There is one project arrival per month $(N=3)$ and the payoffs of the incoming orders are forecast to adhere to a continuous uniform distribution with density function:

$$
\begin{aligned}
u_{Y}(y) & =\frac{1}{12}, \quad 2 \leq y \leq 14 \\
& =0, \quad \text { otherwise }
\end{aligned}
$$

The DP algorithm described by Eq. (3) corresponds with the third-stage values $(k=N)$ :

$$
\begin{array}{ll}
f_{3}((1),(0)) & =y_{3}, \\
E\left[f_{3}((1),(0))\right] & =\int_{2}^{14}\left(\frac{1}{12} y\right) d y=8, \\
f_{3}((0),(0)) & =0 .
\end{array}
$$

For $f_{3}((1),(0))$, we can choose between two order plans: $\mathbf{a}_{3}^{0}$ and $\mathbf{a}_{3}^{1}$. $\mathbf{a}_{3}^{0}$ is the null vector: $\mathbf{0}_{3}=((0),(0))$. Order plan $\mathbf{a}_{3}^{1}=((1),(0))$ implies the allocation of the regular capacity unit from period 3 to the project. Since rejection has a reward of 0 , order plan $\mathbf{a}_{3}^{1}$ maximizes the reward.

The values for stage 2 are the following. We only show the state vectors that will be employed 
in the first stage, and we identify the elements of the control space between straight brackets:

$$
\begin{array}{rll}
f_{2}((1,1),(1,0)) & =\max \left\{\begin{array}{ll}
E\left[f_{3}((1),(0))\right] & {\left[\mathbf{a}_{2}^{0}=\mathbf{0}_{2}\right]} \\
y_{2}+E\left[f_{3}((1),(0))\right] & {\left[\mathbf{a}_{2}^{1}=((1,0),(0,0))\right]} \\
y_{2}+E\left[f_{3}((0),(0))\right] & {\left[\mathbf{a}_{2}^{2}=((0,1),(0,0))\right]} \\
y_{2}-5+E\left[f_{3}((1),(0))\right] & {\left[\mathbf{a}_{2}^{3}=((0,0),(1,0))\right]}
\end{array}\right\}, \\
E\left[f_{2}((1,1),(1,0))\right]=y_{2}+E\left[f_{3}((1),(0))\right], & \\
f_{2}((0,1),(1,0)) & =\int_{2}^{14}\left(\frac{1}{12} y\right) d y+8=16, \\
E\left[f_{2}((0,1),(1,0))\right] & =\int_{2}^{5}\left(\frac{1}{12} \cdot 8\right) d y+\int_{5}^{14} \frac{1}{12}(y+3) d y=11.5, \\
f_{2}((1,0),(1,0)) & =y_{2}, & \\
E\left[f_{2}((1,0),(1,0))\right] & =\int_{2}^{14}\left(\frac{1}{12} y\right) d y=8 .
\end{array}
$$

For $f_{2}((1,1),(1,0))$, the set of eligible order plans contains 4 elements: $\mathcal{A}_{2}=\left\{\mathbf{a}_{2}^{0}, \mathbf{a}_{2}^{1}, \mathbf{a}_{2}^{2}, \mathbf{a}_{2}^{3}\right\}$. Following Lemma 4 , we need not consider order plan $\mathbf{a}_{2}^{2}$ since it is dominated by $\mathbf{a}_{2}^{1}$. Order plan $\mathbf{a}_{2}^{3}$ is dominated by $\mathbf{a}_{2}^{1}$ due to Lemma 1 . The best order plan for $f_{2}((0,1),(1,0))$ cannot be determined unambiguously. If the payoff of the project proposal lies within the interval $[2,5]$, rejection maximizes the expected value of the following stages. If the income lies within $[5,14]$, order plan $\mathbf{a}_{2}^{1}=((0,0),(1,0))$ becomes the best choice.

In the first stage, we have:

$$
\begin{aligned}
f_{1}((0,1,1),(0,1,0)) & =\max \left\{\begin{array}{ll}
E\left[f_{2}((1,1),(1,0))\right] & {\left[\mathbf{a}_{1}^{0}=\mathbf{0}\right]} \\
E\left[f_{2}((0,1),(1,0))\right]+10 & {\left[\mathbf{a}_{1}^{1}=((0,1,0),(0,0,0))\right]} \\
E\left[f_{2}((1,0),(1,0))\right]+10 & {\left[\mathbf{a}_{1}^{2}=((0,0,1),(0,0,0))\right]}
\end{array}\right\}, \\
& =\max \{16 ; 21.5 ; 18\} .
\end{aligned}
$$

Given the example's capacity profile, we can choose from three order plans in the first stage. The first corresponds to rejection; the other two consist of planning in period 2 and 3 , respectively. Since $E\left[f_{2}((0,1),(1,0))\right]>E\left[f_{2}((1,0),(1,0))\right]$, the minimum threshold value for accepting a project at time 0 is $\alpha_{1}=4.5$. The incoming order will preferably be planned in time period 2 .

\subsubsection{Example with precedence relations and non-preemption constraints}

As an illustration, we consider a case similar to the semiconductor fabrication line presented in [41]. When a project $k$ is accepted, it remains in the system for $p_{k}$ periods (due to the manufacturing cycle time) without preemption. Currently (at time 0), a project is offered to the company. The company assesses that this project would generate a payoff of 18 $\left(=y_{1}\right)$ if realized with regular capacity. The project would require two work packages of the company's key resource $\left(p_{1}=2\right)$. Delivery of the project is due within two months $\left(l_{1}=2\right)$. 


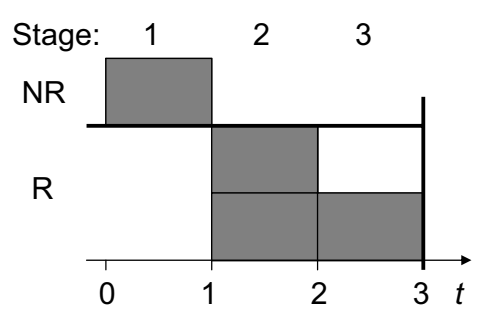

Figure 5: Example with regular (R) and non-regular (NR) capacity units

The problem horizon $T$ is set to three months. At this moment, the profile of the available capacity is $\mathbf{x}_{1}=((0,2,1),(1,0,0))$, a visualization is given in Figure 5 . The interest rate $i$ is set to $1 \%$ per month and the cost of one unit of non-regular capacity amounts to 10 . We anticipate the arrival of one RFQ per month within the problem horizon, so that $N=3$. The company has defined six order types, the average characteristics of which are described by $\mathbf{W}=(P, Y, L)$. The forecasting information contains the following probabilities:

$$
\begin{array}{llll}
\operatorname{Pr}[(1,8,1)] & =0.1, & & \operatorname{Pr}[(1,8,2)]=0.1, \\
\operatorname{Pr}[(1,12,1)]=0.2, & & \operatorname{Pr}[(1,12,2)]=0.1, \\
\operatorname{Pr}[(2,18,2)]=0.1, & & \operatorname{Pr}[(2,16,2)]=0.4 .
\end{array}
$$

We derive the following third-stage values:

$$
\begin{aligned}
& f_{3}((1),(0))= \begin{cases}y_{3}, & \text { if } p_{3}=1, \\
0, & \text { if } p_{3}=2,\end{cases} \\
& E\left[f_{3}((1),(0))\right]=0.1(8+8+12)+0.2 \times 12+0.1 \times 0+0.4 \times 0=5.2, \\
& f_{3}((0),(0))=0 .
\end{aligned}
$$

We calculate $E\left[f_{3}((1),(0))\right]$ as the sum of the probabilities of the different order types multiplied with the corresponding values for $f_{3}((1),(0))$. 
The second-stage computations are:

$$
\begin{aligned}
& f_{2}((2,1),(0,0))= \begin{cases}y_{2}(1.01)+E\left[f_{3}((1),(0))\right], & \text { if } p_{2}=1 \wedge l_{2}=1, \\
\max \left\{y_{2}(1.01)+E\left[f_{3}((1),(0))\right] ; y_{2}\right\}, & \text { if } p_{2}=1 \wedge l_{2}=2, \\
\max \left\{E\left[f_{3}((1),(0))\right] ; y_{2}\right\}, & \text { if } p_{2}=2,\end{cases} \\
& E\left[f_{2}((2,1),(0,0))\right]=0.2(8 \times 1.01+5.2)+0.3(12 \times 1.01+5.2) \\
& +0.1 \times 18+0.4 \times 16=16.052 \text {, } \\
& f_{2}((2,0),(0,0))= \begin{cases}y_{2}(1.01)+E\left[f_{3}((0),(0))\right], & \text { if } p_{2}=1, \\
0, & \text { otherwise, }\end{cases} \\
& E\left[f_{2}((2,0),(0,0))\right]=0.2(8 \times 1.01)+0.3(12 \times 1.01)=5.252, \\
& f_{2}((1,1),(0,0))= \begin{cases}y_{2}(1.01)+E\left[f_{3}((1),(0))\right], & \text { if } p_{2}=1 \wedge l_{2}=1, \\
\max \left\{y_{2}(1.01)+E\left[f_{3}((1),(0))\right] ; y_{2}\right\}, & \text { if } p_{2}=1 \wedge l_{2}=2, \\
\max \left\{E\left[f_{3}((1),(0))\right] ; y_{2}\right\}, & \text { if } p_{2}=2,\end{cases} \\
& E\left[f_{2}((1,1),(0,0))\right]=0.2(8 \times 1.01+5.2)+0.3(12 \times 1.01+5.2) \\
& +0.1 \times 18+0.4 \times 16=16.052 \text {, } \\
& f_{2}((1,0),(0,0))= \begin{cases}y_{2}(1.01)+E\left[f_{3}((0),(0))\right], & \text { if } p_{2}=1, \\
0, & \text { otherwise }\end{cases} \\
& E\left[f_{2}((1,0),(0,0))\right]=0.2(8 \times 1.01)+0.3(12 \times 1.01)=5.252, \\
& f_{2}((0,1),(0,0))= \begin{cases}\max \left\{E\left[f_{3}((1),(0))\right] ; y_{2}\right\}, & \text { if } p_{2}=1 \wedge l_{2}=2, \\
E\left[f_{3}((1),(0))\right], & \text { otherwise, }\end{cases} \\
& E\left[f_{2}((0,1),(0,0))\right]=0.1 \times 8+0.1 \times 12+0.8 \times 5.2=6.16 .
\end{aligned}
$$

The first-stage computations suggest acceptance of the project $\mathbf{w}_{1}$ :

$$
\begin{aligned}
f_{1}((0,2,1),(1,0,0)) & =\max \left\{\begin{array}{ll}
E\left[f_{2}((2,1),(0,0))\right] & {\left[\mathbf{a}_{1}^{0}\right]} \\
E\left[f_{2}((1,1),(0,0))\right]+18 \times 1.01-10 & {\left[\mathbf{a}_{1}^{1}\right]}
\end{array}\right\} \\
& =\max \{16.052 ; 24.232\}
\end{aligned}
$$

In stage one, two order plans are taken into consideration; with the first one being $\mathbf{a}_{1}^{0}=$ $((0,0,0),(0,0,0))$. The second order plan $((0,1,0),(1,0,0))$ allocates the non-regular capacity unit from period 1 at a cost of 10 and the regular capacity unit from period 2. The finish time of the project precedes the end of the planning horizon, $T$, so that interest revenues are reaped. From the stage-one computations we learn that the order can be optimally executed with one non-regular capacity unit from period 1 and one regular capacity unit from the second period. In addition, we are able to formulate an acceptance rule for incoming projects with the same characteristics: for this example, the payoff threshold is 9.901 in combination with order plan $\mathbf{a}_{1}^{1}$. If we changed the cost $c$ of a unit non-regular capacity to 20 , however, it would no longer be optimal to make use of this capacity unit. In this case, $f_{1}((0,2,1),(1,0,0))=\max \{16.052 ; 14.232\}$ and it would be best to reject the offer. In addition, the payoff threshold would be raised to 19.802 . 


\section{Insights and discussion}

In Section 4 we have derived project-specific acceptance thresholds that represent the minimum revenue desired from a project. For the optimal stopping problem (Section 4.1), on the other hand, the threshold is actually the minimum bid required for the sale of one specific capacity unit. While elaborating this basic model, we have in fact determined minimum prices for specific combinations of capacity units as specified by the order plans. The best combination of capacity units led us to a payoff threshold for the incoming project. Based on the threshold calculation in Section 4.2 (and contrary to the models of Bertsekas), one can easily construct examples where the threshold for a specific project exhibits an irregular evolution in the stage number.

The presented SDP approach has the advantage of easily dealing with many different problem characteristics (varying due dates, hiring non-regular capacity units, precedence relations,...). A downside to this high flexibility is the large problem size, reflected in the numerous variables in the state vector. As is typical for dynamic programming, our SDP also suffers from the curse of dimensionality. Nevertheless, important insights can be gained from studying the SDP recursion in Eq. (3). The overall lesson to be learnt is that optimal acceptance and planning decisions depend on (1) the immediate reward as well as (2) the expected future rewards. The immediate reward can easily be maximized, while high expected future rewards are the result of a good fit between the future arrival characteristics and the capacity profile (after the stage's capacity allocation). Unfortunately, optimal 'short-cut' rules cannot easily be determined, and may not even exist, since the influential characteristics have diverging effects. For example, different properties of the order arrival stream (e.g. finite maximum time lags) lead to better results in combination with different capacity profiles (e.g. levelled profile).

Using an analytical framework, we isolate and quantify the different influential effects. In our analysis we determine the specific circumstances under which 'simple' planning rules are optimal. In the remainder of this section, we first outline the analytical framework that will be used to examine the influential characteristics. Subsequently, we separate and quantify the effects from perishable capacity units, finite time lags and reinvestment revenues. We also estimate the merit of having a non-regular capacity unit at one's disposal and quantify the value of due-date flexibility. Finally, we examine the introduction of tardiness penalties. 


\subsection{Analytical framework}

We consider an analytical framework with single-sized incoming projects of order type $A$ and $B$. Order type $z$ has a maximum time lag of $l_{z}$ and a payoff of $y_{z}, z=A, B$; we assume that $y_{A}>y_{B}$. The probability that an arrival belongs to type $z$ is denoted as $\operatorname{Pr}[z]$. We consider the capacity profile $\mathbf{x}_{1}=((0, \ldots, 1,1),(0, \ldots, 0,1))$. Within our framework, periods coincide with stages. The cost of a non-regular capacity unit may vary between 0 and $\infty$, and we distinguish three cases:

(a) $c=0$ : the available overtime is free;

(b) $0<c<\infty$ : overtime can be hired at a certain cost;

(c) $c=\infty$ : there are no overtime opportunities.

These three cases are visualized in Figure 6.

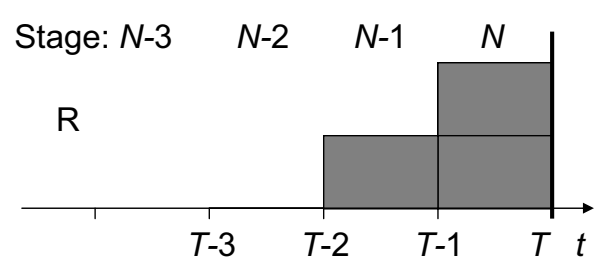

(a) $c=0$

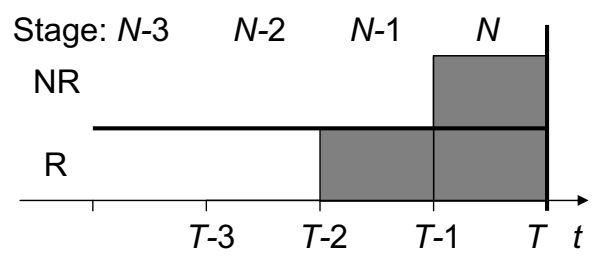

(b) $c>0$

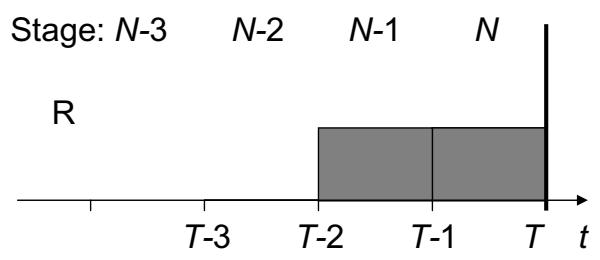

(c) $c=\infty$

Figure 6: Analytical framework with regular (R) and non-regular (NR) capacity units

The stage- $N$ values are derived for the general case:

$$
\begin{aligned}
& E\left[f_{N}((1),(1))\right]=\operatorname{Pr}[A] \cdot y_{A}+\operatorname{Pr}[B] \cdot y_{B}=\bar{y} \\
& E\left[f_{N}((0),(1))\right]=\operatorname{Pr}[A] \cdot \max \left\{y_{A}-c ; 0\right\}+\operatorname{Pr}[B] \cdot \max \left\{y_{B}-c ; 0\right\}
\end{aligned}
$$

Using the recursion from Eq. (3) and invoking Lemma 1 we obtain the expected values for 
stage $(N-1)$ :

$$
\begin{aligned}
E\left[f_{N-1}((1,1),(0,1))\right]= & \bar{y}(2+i), \\
E\left[f_{N-1}((1,0),(0,1))\right]= & \bar{y}(1+i)+\sum_{z=A, B} \operatorname{Pr}[z] \cdot \max \left\{y_{z}-c ; 0\right\}, \\
E\left[f_{N-1}((1,0),(0,0))\right]= & \bar{y}(1+i), \\
E\left[f_{N-1}((0,1),(0,1))\right]= & \sum_{z=A, B} \operatorname{Pr}[z] \cdot \max \left\{\text { if } l_{z}>1: y_{z}+E\left[f_{N}((0),(1))\right], \text { else } 0 ; \bar{y}\right\}, \\
E\left[f_{N-1}((0,0),(0,1))\right]= & \operatorname{Pr}[A] \cdot \max \left\{\text { if } l_{A}>1: y_{A}-c, \text { else } 0 ; \operatorname{Pr}[A] \cdot \max \left\{y_{A}-c ; 0\right\}\right. \\
& \left.+\operatorname{Pr}[B] \cdot \max \left\{y_{B}-c ; 0\right\}\right\}+\operatorname{Pr}[B] \cdot\left(\operatorname{Pr}[A] \cdot \max \left\{y_{A}-c ; 0\right\}+\right. \\
& \left.\operatorname{Pr}[B] \cdot \max \left\{y_{B}-c ; 0\right\}\right) .
\end{aligned}
$$

For stage $(N-2)$, we calculate:

$$
\begin{aligned}
E\left[f_{N-2}((0,1,1),(0,0,1))\right]= & \sum_{z=A, B} \operatorname{Pr}[z] \cdot \max \left\{\text { if } l_{z}>2: y_{z}+E\left[f_{N-1}((1,0),(0,1))\right],\right. \\
& \text { else } 0 ; \text { if } l_{z}>1: y_{z}(1+i)+E\left[f_{N-1}((0,1),(0,1))\right], \text { else 0; } \\
& \bar{y}(2+i)\}, \\
E\left[f_{N-2}((0,1,0),(0,0,1))\right]= & \sum_{z=A, B} \operatorname{Pr}[z] \cdot \max \left\{\text { if } l_{z}>2: y_{z}-c+\right. \\
& E\left[f_{N-1}((1,0),(0,0))\right], \text { else } 0 ; \text { if } l_{z}>1: y_{z}(1+i) \\
& \left.+E\left[f_{N-1}((0,0),(0,1))\right], \text { else } 0 ; E\left[f_{N-1}((1,0),(0,1))\right]\right\}, \\
E\left[f_{N-2}((0,0,1),(0,0,1))\right]= & \sum_{z=A, B} \operatorname{Pr}[z] \cdot \max \left\{\text { if } l_{z}>2: y_{z}+E\left[f_{N-1}((0,0),(0,1))\right],\right. \\
& \text { else } \left.0 ; E\left[f_{N-1}((0,1),(0,1))\right]\right\} .
\end{aligned}
$$

The foregoing quantities will be used throughout the remainder of Section 5 .

\subsection{Perishable capacity units}

In accordance with the analytical framework, we will say that early planning, meaning planning on the first available capacity unit, is preferred in stage $(N-k)$ if

$y_{N-k}(1+i)+E\left[f_{N-k-1}((0, \ldots, 0,1),(0, \ldots, 0,1))\right] \geq y_{N-k}+E\left[f_{N-k-1}((0, \ldots, 1,0),(0, \ldots, 0,1))\right]$.

We obtain the following result:

Theorem 1. Within the analytical framework, in the absence of reinvestment revenues $(i=$ 0 ) and in case of infinite time lags $\left(l_{z}=\infty, z=A, B\right)$, early planning is preferred if the cost of a non-regular capacity unit is larger than $y_{B}$, otherwise the planner remains indifferent to the chosen order plan.

The proof appears in the appendix. Logically, early available capacity units cannot give rise to more allocation opportunities than later ones and thus have a larger risk of perishing without being used.

Previously, we have shown in Lemma 4 and Corollary 1 that order plans that allocate current capacity units are favored. Likewise, Theorem 1 suggests that in the absence of reinvestment revenues and in case of infinite time lags, we need only consider order plans that plan early in time. 


\subsection{Finite maximum time lags}

When we restrict the length of the maximum time lags, Theorem 1 no longer applies. The reason is that capacity units from early time periods do not necessarily generate fewer allocation opportunities than units from later periods. As an example we present rush orders, which we define to be order types with higher payoffs and smaller time lags than other order types.

A rush order with a maximum time lag of one can only be planned at its release time, so that, for a specific capacity unit, the probability of being allocated to this rush order depends on the number of available capacity units in the same period and not on the time until the unit perishes. Under these circumstances, planning other order types on the early capacity unit may no longer be the best option. This intuition is confirmed by our analysis based on the analytical framework from Section 5.1. Suppose that order type $A$ is a rush order, so that $y_{A}>y_{B} \wedge l_{A}<l_{B}$. For convenience, we set $l_{A}=1, l_{B}>3$ and $i=0$ to eliminate the influence of reinvestment revenues. From the analytical framework, we learn that early planning does not prevail for cases (a) and (b) since

$$
E\left[f_{N-2}((0,0,1),(0,0,1))\right]<E\left[f_{N-2}((0,1,0),(0,0,1))\right] .
$$

This is because the resulting profile on the right side of the equation has more opportunities to accept high-payoff rush orders. In case (c), both options are equally good as a consequence of equal opportunities for accepting project $A$ in both resulting capacity profiles. These equations remain valid for offers in stage $(N-4)$.

Building on the foregoing paragraph, we advance that companies that are confronted with rush orders benefit from a levelled capacity profile, which is a profile with more or less equal allocation opportunities in every period. This can be illustrated through a comparison of the profits resulting from a non-levelled profile $\mathbf{x}_{N-2}^{N L}=((0,0,2)(0,0,0))$ and a levelled profile $\mathbf{x}_{N-2}^{L}=((0,1,1)(0,0,0))$. In the presence of a rush order type $A$ with $l_{A}=1$ (and $\left.i=0\right)$, it holds that $E\left[f_{N-2}\left(\mathbf{x}_{N-2}^{L}\right)\right]>E\left[f_{N-2}\left(\mathbf{x}_{N-2}^{N L}\right)\right]$, which implies that the levelled profile leads to better results, whereas the company would remain neutral in relation to both profiles if the time lags of both types were sufficiently large.

\subsection{Reinvestment revenues}

When the interest rate $i$ is strictly positive, reinvestment revenues can be reaped. In stage $(N-3)$ of the analytical framework, early planning is best in all three cases from the 
analytical framework when

$$
y_{N-3}>\operatorname{Pr}[A] \cdot y_{A}+\operatorname{Pr}[B] \cdot \bar{y} .
$$

In addition, early planning is preferred if $c \geq y_{B}$ for very small interest rates. For incoming projects in stage $(N-4)$, arrival $\mathbf{w}_{N-4}$ is planned early if

$$
y_{N-4}>\operatorname{Pr}[A] \cdot y_{A}+\operatorname{Pr}[B] \cdot\left(\operatorname{Pr}[A] \cdot y_{A}+\operatorname{Pr}[B] \cdot \bar{y}\right)
$$

Early planning is also best when $c \geq y_{B}$ and the interest rate is near zero. The righthand side of the two foregoing equations constitutes a cut-off value on the payoff of the incoming project, above which early planning is preferred; one could speak of high-versus low-payoff projects. We conclude that, when the interest rate $i$ has a positive value, high payoffs generate high reinvestment revenues and are thus better planned early in time, while low-payoff projects are pushed later in time to leave room for other projects.

We also observe that the cut-off value for early planning increases with the number of stages $N$ in the planning horizon. This observation is related to the fact that the value function of our SDP (as given by Eq. (3)) is non-decreasing with $N$.

\subsection{Non-regular capacity units}

The presented model supports the quantification of the value of having non-regular capacity units at one's disposal. This can be useful when the price of keeping non-regular capacity available needs to be negotiated with subcontractors.

As an example we again consider the rush-order case from Section 5.3. When calculating the difference between the expected value of case (b) and (a), we derive the value of the non-regular capacity unit in stage $(N-3)$. If $y_{B}>c$ then $E\left[f_{N-2}((0,1,1),(0,0,1))\right]-$ $E\left[f_{N-2}((0,1,1),(0,0,0))\right]=\operatorname{Pr}[B] \cdot\left(y_{B}-c\right)$ and if $y_{B} \leq c$ then $E\left[f_{N-2}((0,1,1),(0,0,1))\right]-$ $E\left[f_{N-2}((0,1,1),(0,0,0))\right]=0$, so that the availability of a non-regular capacity unit is valuable only if the unit hiring cost is smaller than the payoff of project $B$. In this setting, the value of the non-regular capacity unit is independent of $y_{A}$, which may seem counterintuitive. The reason is simply that the rush order under examination can only be executed at its release time so that only orders of type $B$ can be associated with the non-regular capacity unit in question. 


\subsection{Valuation of flexible due dates}

Our model also allows for the valuation of increased flexibility under the form of an extension of the maximum time lag. When the due date of an incoming project $\mathbf{w}_{k}$ is increased, $f_{k}$ changes to $f_{k}^{\prime}$. The value of the increase in flexibility is equal to $\Omega=f_{k}^{\prime}-f_{k}$. Since the set $\mathcal{A}_{k}$ of eligible actions for $f_{k}$ is a subset of the set $\mathcal{A}_{k}^{\prime}$ of eligible actions for $f_{k}^{\prime}, \Omega$ is larger than or equal to $0 . \Omega$ is strictly positive only if the extension of the due date gives rise to new order plans (if $\mathcal{A}_{k}^{\prime} \backslash \mathcal{A}_{k} \neq \emptyset$ ), and we consider only such cases. We see that $\Omega>0$ if, for $y_{k}(k=1, \ldots, N)$, there exists a new order plan $\hat{j}, \hat{j} \in\left(\mathcal{A}_{k}^{\prime} \backslash \mathcal{A}_{k}\right)$, for which $f_{k}^{\hat{j}}\left(\mathbf{x}_{k}\right)$, as defined in Section 4.2 , is strictly larger than $f_{k}^{\tilde{j}}\left(\mathbf{x}_{k}\right)$, for any $\tilde{j} \in \mathcal{A}_{k}$. If $i=0$, the slope of $f_{k}^{j}$ is 0 , otherwise the slope of $f_{k}^{j}$ for $j>0$ equals $(1+i)^{\left(T-t_{j}^{*}\right)} \geq 1$. The slopes for the new order plans are strictly smaller than the slopes for the existing order plans (for $j \neq 0$ ), due to the larger realization times of the new order plans. The intercept of $f_{k}^{j}$ consists of the costs of non-regular capacity (if $k=N$ ) added to the expected reward $E\left[f_{k+1}\right]$ from future stages (if $k \neq N$ ).

We conclude that increased due-date flexibility has a strictly positive value if at least one new order plan offers a better combination of non-regular capacity usage and resulting capacity. When interest rates are strictly positive and acceptance was previously impossible, flexibility pays off when the project's revenue is sufficiently large. The value of the increase in flexibility, $\Omega$, represents the increase in the expectation at time 0 of the value at time $T$ of the selected portfolio. When using this value during negotiations with the customer (e.g. for granting discounts), the time value of money should obviously be taken into account.

\subsection{Tardiness penalties}

The reward function in Eq. (2) can easily be adapted for the presence of tardiness penalties. In effect, rather than fixed, unconditional deadlines, this corresponds with the common case where due dates can be violated, be it at a (known) penalty. When the due date $d_{k}$ of a project is violated, the payoff the company would receive at completion time is reduced by a tardiness cost $\tilde{T}_{j}^{*}$ so that the reward function changes to

$$
g_{k}\left(\mathbf{a}_{k}^{j}, \mathbf{w}_{k}\right)= \begin{cases}0 & \text { if } j=0, \\ \left(y_{k}-\tilde{T}_{j}^{*}\right)(1+i)^{\left(T-t_{j}^{*}\right)}-c s_{j}^{*} & \text { otherwise. }\end{cases}
$$

If we assume that the tardiness cost increases linearly with the length of the due-date violation, then $\tilde{T}_{j}^{*}=\max \left\{0 ; \tilde{c}\left(t_{j}^{*}-d_{k}\right)\right\}$, with $\tilde{c}$ the per period due-date violation penalty. Since 
the tardiness cost for any specific order plan is a constant value, it is easy to see that Lemma 1, 2, 3, 4 and Corollary 1 remain valid when tardiness penalties apply.

\section{Conclusions and further research}

In this paper we have investigated dynamic order acceptance and planning in an overdemanded multi-project organization that aims at maximizing its profits. We have stressed the importance of integrating order acceptance and capacity planning in order to be able to live up to competitive due dates and reduce the sometimes excessive use of highly expensive non-regular capacity. We have used stochastic dynamic programming to maximize the expected profits of the company within the planning horizon. Our exact methods have allowed us to gain valuable insights into how the problem characteristics influence the acceptance and capacity-planning decisions. We have separated and quantified the influence of a number of problem characteristics, such as perishable capacity units, finite maximum time lags, reinvestment revenues and non-regular capacity units.

We have established that without reinvestment revenues and with infinite deadlines, one need only consider early planning. When the maximum time lags become restrictive, this policy is no longer optimal; when companies are confronted with rush orders, for instance, it makes more sense to strive for a levelled capacity profile. In general, the best planning policy aims at reducing the risk of having to reject short-lagged projects because of a lack of available capacity units. The effect of reinvestment revenues on the planning decision has also been investigated. We conclude that high payoffs generate high reinvestment revenues and are thus better planned early; our model allows us to determine the cut-off between high- and low-payoff projects. In the final paragraphs, we have quantified the gains from non-regular capacity units and from due-date flexibility and we have introduced tardiness penalties.

The problems that we can solve to optimality remain limited in size, and so further research is needed into producing planning solutions for real-life problems. We are convinced that the model and insights described in this paper can serve as guidelines in this process. 


\section{Appendix: proofs}

Proof (Theorem 1): The theorem is proven by induction on the number of stages. As initial step, we wish to show that

$$
E\left[f_{N-2}((0,0,1),(0,0,1))\right] \geq E\left[f_{N-2}((0,1,0),(0,0,1))\right]
$$

Using the analytical framework, it is easy to establish that:

$$
\begin{aligned}
& \text { If } c \leq y_{B} \Rightarrow E\left[f_{N-2}((0,0,1),(0,0,1))\right]=E\left[f_{N-2}((0,1,0),(0,0,1))\right] \\
& \text { otherwise } \Rightarrow E\left[f_{N-2}((0,0,1),(0,0,1))\right]>E\left[f_{N-2}((0,1,0),(0,0,1))\right] .
\end{aligned}
$$

The second part of the proof consists in demonstrating the validity of the induction step: $E\left[f_{N-k}((0, \ldots, 0,1),(0, \ldots, 0,1))\right] \geq E\left[f_{N-k}((0, \ldots, 1,0),(0, \ldots, 0,1))\right] \Rightarrow$ $E\left[f_{N-k-1}((0, \ldots, 0,1),(0, \ldots, 0,1))\right] \geq E\left[f_{N-k-1}((0, \ldots, 1,0),(0, \ldots, 0,1))\right]$.

The induction step can be written as:

$$
\begin{aligned}
& \sum_{z=A, B} \operatorname{Pr}[z] \cdot \max \left\{\begin{array}{ll}
E\left[f_{N-k+1}((0, \ldots, 0,1),(0, \ldots, 0,1))\right] & {\left[\mathbf{a}_{N-k}^{0}\right]} \\
y_{z}+E\left[f_{N-k+1}((0, \ldots, 0,0),(0, \ldots, 0,1))\right] & {\left[\mathbf{a}_{N-k}^{1}\right]}
\end{array}\right\} \geq \\
& \sum_{z=A, B} \operatorname{Pr}[z] \cdot \max \left\{\begin{array}{ll}
E\left[f_{N-k+1}((0, \ldots, 1,0),(0, \ldots, 0,1))\right] & {\left[\tilde{\mathbf{a}}_{N-k}^{0}\right]} \\
y_{z}+E\left[f_{N-k+1}((0, \ldots, 0,0),(0, \ldots, 0,1))\right] & {\left[\tilde{\mathbf{a}}_{N-k}^{1}\right]} \\
y_{z}-c+E\left[f_{N-k+1}((0, \ldots, 1,0),(0, \ldots, 0,0))\right] & {\left[\tilde{\mathbf{a}}_{N-k}^{2}\right]}
\end{array}\right\} \\
& \Rightarrow \\
& \begin{array}{c}
\sum_{z=A, B} \operatorname{Pr}[z] \cdot \max \left\{\begin{array}{ll}
E\left[f_{N-k}((0, \ldots, 0,1),(0, \ldots, 0,1))\right] & {\left[\mathbf{a}_{N-k-1}^{0}\right]} \\
y_{z}+E\left[f_{N-k}((0, \ldots, 0,0),(0, \ldots, 0,1))\right] & {\left[\mathbf{a}_{N-k-1}^{1}\right]}
\end{array}\right\} \geq \\
\sum_{z=A, B} \operatorname{Pr}[z] \cdot \max \left\{\begin{array}{ll}
E\left[f_{N-k}((0, \ldots, 1,0),(0, \ldots, 0,1))\right] & {\left[\tilde{\mathbf{a}}_{N-k-1}^{0}\right]} \\
y_{z}+E\left[f_{N-k}((0, \ldots, 0,0),(0, \ldots, 0,1))\right] & {\left[\tilde{\mathbf{a}}_{N-k-1}^{1}\right]} \\
y_{z}-c+E\left[f_{N-k}((0, \ldots, 1,0),(0, \ldots, 0,0))\right] & {\left[\tilde{\mathbf{a}}_{N-k-1}^{2}\right]}
\end{array}\right\} .
\end{array}
\end{aligned}
$$

Case 1: $c \geq y_{A}$; due to Lemma 3 the allocation of the non-regular capacity unit $\left(\tilde{\mathbf{a}}_{k}^{2}\right)$ need not be considered. The validity of the induction step is based on two arguments. First of all, planning on the regular capacity unit $\left(\mathbf{a}_{k}^{1}\right.$ and $\left.\tilde{\mathbf{a}}_{N-k}^{1}\right)$ results in the same reward $y_{z}+E\left[f_{N-k}((0, \ldots, 0,0),(0, \ldots, 0,1))\right]$ for both capacity profiles. Secondly, the induction hypothesis states that in case of rejection, $E\left[f_{N-k}((0, \ldots, 0,1),(0, \ldots, 0,1))\right]$ is larger than or equal to $E\left[f_{N-k}((0, \ldots, 1,0),(0, \ldots, 0,1))\right]$.

Case 2: $c<y_{A}$; using the argumentation from case 1, the theorem is proven if we demonstrate that $y_{z}+E\left[f_{N-k}((0, \ldots, 0,0),(0, \ldots, 0,1))\right] \geq y_{z}-c+E\left[f_{N-k}((0, \ldots, 1,0),(0, \ldots, 0,0))\right]$. We again consider two cases: $c<y_{B}$ and $c \geq y_{B}$. 
If $c<y_{B}$, it follows that:

$$
\begin{aligned}
& E\left[f_{N-k}((0, \ldots, 0,0),(0, \ldots, 0,1))\right] \geq-c+E\left[f_{N-k}((0, \ldots, 1,0),(0, \ldots, 0,0))\right] \\
& \quad \Rightarrow\left(y_{A}-c\right) \cdot \operatorname{Pr}[A] \cdot\left(1-(\operatorname{Pr}[B])^{k+1}\right) / \operatorname{Pr}[A]+\left(y_{B}-c\right) \cdot(\operatorname{Pr}[B])^{k+1} \geq \\
& \quad-c+y_{A} \cdot \operatorname{Pr}[A] \cdot\left(1-(\operatorname{Pr}[B])^{k}\right) / \operatorname{Pr}[A]+y_{B}(\operatorname{Pr}[B])^{k} \\
& \quad \Rightarrow 0 \geq\left(y_{B}-y_{A}\right) \cdot(\operatorname{Pr}[B])^{k} \cdot \operatorname{Pr}[A] .
\end{aligned}
$$

If $c \geq y_{B}$, we do not accept project $B$ because of Lemma 3, so that

$$
\begin{aligned}
& E\left[f_{N-k}((0, \ldots, 0,0),(0, \ldots, 0,1))\right] \geq-c+E\left[f_{N-k}((0, \ldots, 1,0),(0, \ldots, 0,0))\right] \\
& \quad \Rightarrow\left(y_{A}-c\right) \cdot \operatorname{Pr}[A] \cdot\left(1-(\operatorname{Pr}[B])^{k+1}\right) / \operatorname{Pr}[A] \geq-c+y_{A} \cdot \operatorname{Pr}[A] \cdot\left(1-(\operatorname{Pr}[B])^{k}\right) / \operatorname{Pr}[A] \\
& \quad \Rightarrow \operatorname{Pr}[B] \cdot c \geq y_{B}-y_{A} \cdot \operatorname{Pr}[A] .
\end{aligned}
$$

\section{References}

[1] P.S. Adler, A. Mandelbaum, V. Nguyen, and E. Schwerer. From project to process management: an empirically-based framework for analyzing product development time. Management Science, 41(3):458-484, 1995.

[2] C. Akkan. Finite-capacity scheduling-based planning for revenue-based capacity management. European Journal of Operational Research, 100:170-179, 1997.

[3] N. Baker and J. Freeland. Recent advances in R\&D benefit measurement and project selection methods. Management Science, 21(10):1164-1175, 1975.

[4] N. Balakrishnan, J.W. Patterson, and S.V. Sridharan. Rationing capacity between two product classes. Decision Sciences, 27(2):185-214, 1996.

[5] J.F. Bard, R. Balachandra, and P.E. Kaufman. An interactive approach to R\&D project selection and termination. IEEE Transactions on Engineering Management, 35(3):139$146,1988$.

[6] G.J. Beaujon, S.P. Martin, and G.C. McDonald. Balancing and optimizing a portfolio of R\&D projects. Naval Research Logistics, 48(1):18-40, 2001.

[7] R. Bellman. Dynamic programming. Princeton University Press, Princeton, NJ, 1957.

[8] D.P. Bertsekas. Dynamic programming and optimal control, volume 1. Athena Scientific, 2005. 
[9] M.S. Brenner. Practical R\&D project prioritization. Research Technology Management, 37(5):38-42, 1994.

[10] R.G. Cooper, S.J. Edgett, and E.J. Kleinschmidt. New product portfolio management: Practices and performance. Journal of Product Innovation Management, 16(4):333-351, 1999.

[11] A.F. Czajkowski and S. Jones. Selecting interrelated R\&D projects in space planning technology. IEEE Transactions on Engineering Management, 33(1):17-24, 1986.

[12] P. De, J.B. Ghosh, and C.E. Wells. Job selection and sequencing on a single machine in a random environment. European Journal of Operational Research, 70:425-431, 1993.

[13] R. De Boer. Resource-constrained multi-project management - A hierarchical decision support system. PhD thesis, University of Twente, Enschede, the Netherlands, 1998.

[14] B. De Reyck. Scheduling projects with generalized precedence relations. Exact and heuristic procedures. $\mathrm{PhD}$ thesis, Department of applied economics, Katholieke Universiteit Leuven, 1998.

[15] E.L. Demeulemeester and W.S. Herroelen. Project scheduling - A research handbook. Kluwer Academic Publishers, Dordrecht, 2002.

[16] M.W. Dickinson, A.C. Thornton, and S. Graves. Technology portfolio management: optimizing interdependent projects over multiple time periods. IEEE Transactions on Engineering Management, 48(4):518-527, 2001.

[17] A.K. Dixit and R.S. Pindyck. Investment under uncertainty. Princeton University Press, 1994.

[18] M.J. Ebben, E.W. Hans, and F.M. Olde Weghuis. Workload based order acceptance in job shop environments. OR Spektrum, 27:107-122, 2005.

[19] H. Eilat, B. Golany, and A. Shtub. Constructing and evaluating balanced portfolios of R\&D projects with interactions: a DEA based methodology. European Journal of Operational Research, 172:1018-1039, 2006.

[20] G.E. Fox, N.R. Baker, and L.J. Bryant. Economic models for R\&D project selection in the presence of project interactions. Management Science, 30:890-902, 1984. 
[21] N. Gademann and M. Schutten. Linear-programming-based heuristics for project capacity planning. IIE Transactions, 37:153-165, 2005.

[22] K. Golabi. Selecting a group of dissimilar projects for funding. IEEE Transactions on Engineering Management, 34(3):138-145, 1987.

[23] D.L. Hall and A. Nauda. An interactive approach for selecting R\&D projects. IEEE Transactions on Engineering Management, 37:126-133, 1990.

[24] E. Hans. Resource loading by branch-and-price techniques. PhD thesis, University of Twente, Enschede, the Netherlands, 2001.

[25] A.D. Henriksen and A.J. Traynor. A practical R\&D project-selection scoring tool. IEEE Transactions on Engineering Management, 46(2):158-170, 1999.

[26] J. Herbots, W. Herroelen, and R. Leus. Dynamic order acceptance and capacity planning within a multi-project environment. Technical Report KBI 0614, Department of Decision Sciences and Information Management, Katholieke Universiteit Leuven, Belgium, 2006.

[27] W. Herroelen, P. Van Dommelen, and E. Demeulemeester. Project network models with discounted cash flows: A guided tour through recent developments. European Journal of Operational Research, 100:97, 1997.

[28] T. Jørgensen. Project scheduling as a stochastic dynamic decision problem. PhD thesis, Norwegian university of Science and Technology, Trondheim, Norway, 1999.

[29] S. Kavadias and C.H. Loch. Project selection under uncertainty. Dynamically allocating resources to maximize value. Kluwer, 2004.

[30] H. Kerzner. Project management: a systems approach to planning, scheduling, and controlling. John Wiley \& Sons, 1997.

[31] T. Kis. A branch-and-cut algorithm for scheduling of projects with variable-intensity activities. Mathematical Programming, 103(3):515-539, 2005.

[32] A.J. Kleywegt and J.D. Papastavrou. The dynamic and stochastic knapsack problem. Operations Research, 46(1):17-35, 1998. 
[33] A.J. Kleywegt and J.D. Papastavrou. The dynamic and stochastic knapsack problem with random sized items. Operations Research, 49(1):26-41, 2001.

[34] R. Kolisch and K. Meyer. Perspectives in modern project scheduling, chapter Selection and scheduling of pharmaceutical research projects, pages 321-344. Springer, 2006.

[35] N. Levy and S. Globerson. Improving multiproject management by using a queuing theory approach. Project Management Journal, 28(4):40-46, 1997.

[36] H.F. Lewis and S.A. Slotnick. Multi-period job selection: Planning work loads to maximize profit. Computers \& Operations Research, 29:1081-1098, 2002.

[37] M.E. Lewis, H. Ayhan, and R.D. Foley. Bias optimality in a queue with admission control. Probability in the Engineering and Informational Sciences, 13:309-327, 1999.

[38] C.H. Loch and K. Bode-Greuel. Evaluating growth options as sources of value for pharmaceutical research projects. RED Management, 31(2):232-248, 2001.

[39] C.H. Loch, M.T. Pich, C. Terwiesch, and M. Urbschat. Selecting R\&D projects at BMW: A case study of adopting mathematical programming models. IEEE Transactions on Engineering Management, 48(1):70-80, 2001.

[40] D.G. Luenberger. Investment science. Oxford University Press, 1998.

[41] T.C. Perry and J.C. Hartman. Allocating manufacturing capacity by solving a dynamic, stochastic multiknapsack problem. Technical Report ISE 04T-009, Lehigh University, PA, 2004 .

[42] J.F. Ringuest, S.B. Graves, and R.H. Case. Mean-gini analysis in R\&D portfolio selection. European Journal of Operational Research, 154:157-169, 2004.

[43] K. Ross and D. Tsang. The stochastic knapsack problem. IEEE Transactions on Communications, 37(7):740-747, 1989.

[44] S.M. Ross. Introduction to stochastic dynamic programming. Academic Press, Inc, 1983.

[45] T.L. Saaty. How to make a decision: the analytic hierarchy process. Interfaces, 24(6):1943, 1994. 
[46] S.A. Slotnick and T.E. Morton. Selecting jobs for a heavily loaded shop with lateness penalties. Computers \& Operations Research, 23:131-140, 1996.

[47] L.R. Weatherford and S.E. Bodily. A taxonomy and research overview of perishableasset revenue management: Yield management, overbooking and pricing. Operations Research, 40(5):831-844, 1992.

[48] R. Weber, B. Werners, and H.J. Zimmerman. Planning models for research and development. European Journal of Operations Research, 48:175-188, 1990.

[49] F.A. Wester, J. Wijngaard, and W.H. Zijm. Order acceptance strategies in a productionto-order environment with setup times and due-dates. International Journal of Production Research, 30(6):1313-1326, 1992.

[50] S.C. Wheelwright and K.B. Clark. Creating project plans to focus product development. Harvard Business Review, pages 70-82, March-April 1992.

[51] K.-K. Yang and C.-C. Sum. An evaluation of due date, resource allocation, project release, and activity scheduling rules in a multi-project environment. European Journal of Operational Research, 103:139-154, 1997.

[52] W.H. Zijm. Towards intelligent manufacturing planning and control systems. OR Spektrum, 22:313-345, 2000. 